\title{
Surface Stress Induced by Adatoms Adsorption on Solid Surfaces
}

\author{
Zhen-Yu Yang, Zhi-Qiao Wang and Ya-Pu Zhao*
}

\author{
State Key Laboratory of Nonlinear Mechanics (LNM), Institute of Mechanics, Chinese \\ Academy of Sciences, Beijing 100080, China
}

\begin{abstract}
Based on the statistical thermodynamics theory, a theoretical model of adsorbate induced surface stress of adatoms adsorption on solid surface is presented. For the low coverage, the interaction between the adsorbed molecules is entirely negligible and the adsorption induced surface stress is found to be the function of the coverage and the adsorption energy change with strain. For the high coverage, the adsorbate-adsorbate interaction contributes to the adsorption-induced surface stress effectively. In the case of carbon adsorption on the $\mathrm{Ni}(100)$ surface, the value of 0.5 is obtained as a characteristic coverage to decide whether to take the interaction between the adsorabtes into consideration and the results also show that the adsorption induces a compressive surface stress.
\end{abstract}

Keywords: Adsorption, Adatom, Surface stress, Coverage, Adsorption energy

\section{Introduction}

The adsorption induced surface stress has important applications in controlling the materials behavior, such as sensors, molecular devices and other functional structures [1,2]. The physics of solid surfaces are greatly affected by adsorbed atoms or molecules. For example, the frequency of microcantilever-based biosensors is significantly influenced by the surface stress induced by bio-molecules adsorption [3,4]. Further, Ren and Zhao et al. [5] presented direct evidence for the effect of local stress at a micro-cantilever surface on the flexural deformation, which is due to the change of the density of the hydrogen proton.

Many researchers have studied the dependence of surface stress on adatoms concentration [6-8]. Lau and Kohn [9,10] founded that the interaction was repulsive between two identical adsorbates on a surface and varied as $r^{-3}$ with separation $r$. The elastic field induced by an adatom was approximated by a force dipole on the surface of a half-space and adatoms interact through their elastic fields. A continuum-level point-defect model of adatoms with strain-dependent properties was developed by Kukta et al. [11]. Based on continuum theory, the relationship between adatoms and macroscopic stress was deduced [12]. With the linear elastic theory, Pala et al. [13] demonstrated a model to determine the adsorptive and catalytic properties of strained metal surfaces. The solid surface is to some extent deformable, the surface bulges slightly towards the adsorbed molecule, but when the distances are closer the situation is reversed [14]. In the other methods, the interaction between the adsorbates and the substrate in the physisorption was considered as a body force acting on the surface, which gave smaller dimples [15]. The surface Green function for soft elastic half-space was derived by $\mathrm{He}$ et al. [16] ignoring the surface stress effect, which was adopted to analyze the interaction between two adsorbed molecules.

A nonlinear functional form of the adsorbate-induced surface stress dependence on the coverage was proposed by Ibach [17] and was subsequently improved with considering the heat of adsorption [18]. Wang and Xie et al.

*Author to whom any correspondence should be addressed. E-mail: yzhao@imech.ac.cn (Ya-Pu Zhao). 
$[19,20]$ established the theoretical model of the adsorbate-induced surface stress on anion adsorption on $\mathrm{Au}(111)$ surface from the statistical thermodynamics, with the lattice gas model. The relaxation of substrate induced by a chemisorbed molecule was studied theoretically [21], using the quadratic expansion of the potential energy of a free surface. The adsorbate-adsorbate interaction mediated by substrate lattice was calculated [22], assuming that the adsorbate introduces local perturbations around the adsorption site. Many experimental and theoretical researches were carried out to investigate the surface properties of the different surfaces with various adsorptions [23-28]. In addition, the adsorbate-induced stresses of $\mathrm{O} / \mathrm{C}$ adsorption on $\mathrm{Ni}(100)$ were calculated at the density-functional theory (DFT) level [29].

The adsorption induced surface stress, which is due to submonolayer coverages of adsorbates on a substrate, can be measured by experiments now. But the present theoretical models are still not comprehensive enough to fit the various adsorption phenomena to a certain extent or too complicated to be applied in theoretical research. This article focuses on the establishment of a general theoretical model for the adatoms induced surface stress based on the thermodynamics. The contribution to the adsorption induced surface stress includes the adsorption energy, surface coverages and interaction between the adatoms. The relationships between the adsorption induced surface stresses and adsorbate-adsorbate interaction are also analyzed in this work. The substrate in this article is considered to be an isotropic material.

\section{Theoretical model}

For a surface with adsorbates, the electronic structure of the surface may be perturbed by the adsorbed atoms or molecules and the substrate atoms in the surface layers can feel the force exerted by adsorbates, which will change the surface energy as well as surface stress. The change of the stress is called adsorption-induced surface stress. A qualitative distinction is usually made between physisorption and chemisorption (Fig. 1). The physisorption results from the presence of van der Waals attractive forces due to fluctuating dipole moments on the interacting adsorbate and substrate with no charge transfer or electrons shared between atoms. The adsorption energy $\triangle E_{\mathrm{P}}$ is on the order of $50 \sim 500 \mathrm{meV} / \mathrm{atom}$. The chemisorption involves strong bonds and charge transfer, with the adsorption energy $\triangle E_{C}$ of the adatom is of a few eV/atom.

Actually, no absolutely sharp distinction can be made and intermediate cases exist. Based on the theory of thermodynamics, we try to establish a simple model which can be applied to both physisorption and chemisorption.

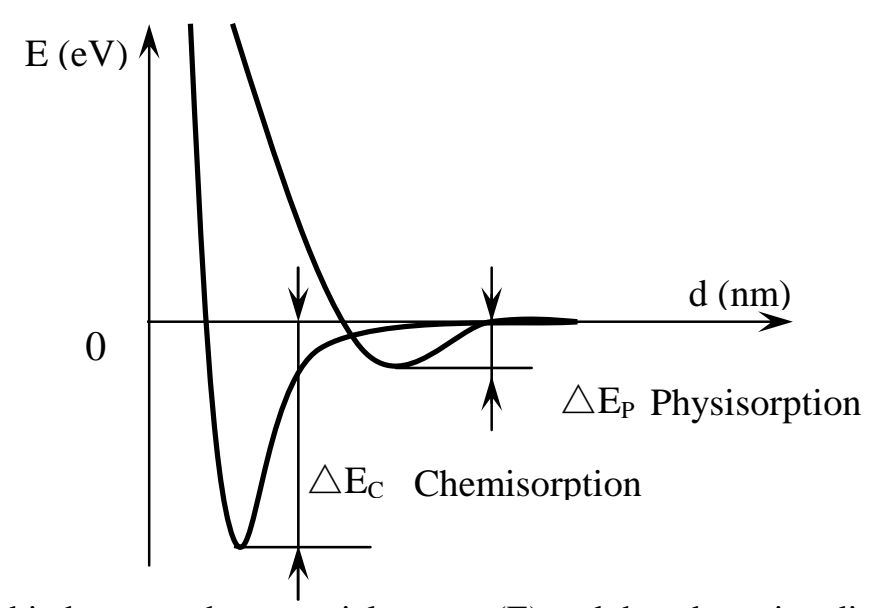

Figure 1. The relationship between the potential energy (E) and the adsorption distances (d). 


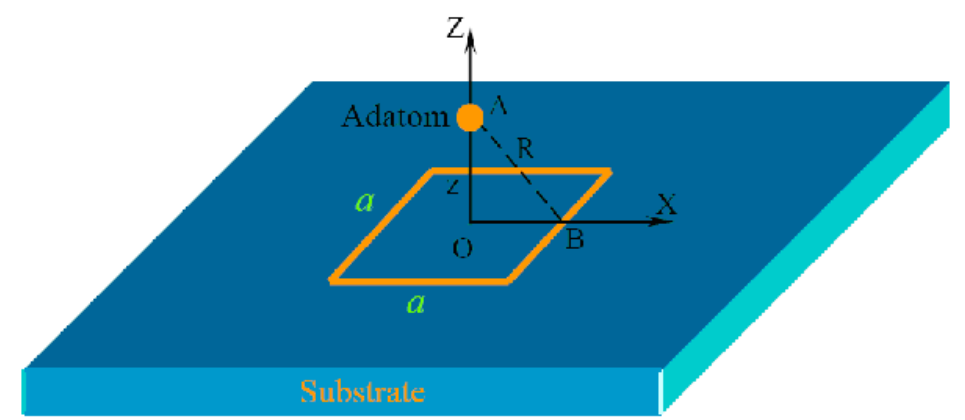

Figure 2. The adatom adsorption on the surbstrate.

\subsection{Adsorption induced surface stress}

Based on the thermal equilibrium conditions in the solid surface, the Gibbs-Duhem equation for the interface can be obtained as [30]

$$
d \gamma=-\frac{S_{s}}{A} d T+\delta \varepsilon(\boldsymbol{\tau}-\gamma \boldsymbol{I})-\Gamma_{i} d \mu_{i}
$$

where $\gamma$ is the surface energy, $S_{s}$ is the surface entropy, $A$ is the surface area, $\boldsymbol{\varepsilon}$ is the surface strain tensor, $\boldsymbol{\tau}$ is the surface stress tensor, $\boldsymbol{I}$ is the second-order unit tensor, $\Gamma_{i}=N_{a} / A$ is the interface density of number of particles $i$, and $\mu_{i}$ is the chemical potential of adsorbate.

From Eq. (1), we can obtain the following two relations

$$
\begin{aligned}
& \left.\frac{\partial \gamma}{\partial \mu_{i}}\right|_{T, \varepsilon}=-\Gamma_{i}, \\
& \left.\frac{\partial \gamma}{\partial \boldsymbol{\varepsilon}}\right|_{T, \mu_{i}}=\boldsymbol{\tau}-\gamma \boldsymbol{I} .
\end{aligned}
$$

Equation (2) is the Gibbs adsorption isotherm and the Eq. (3) is known as the Shuttleworth relationship, which connects the interface stress to the interface energy [31]. Combining the Eqs. (2) and (3), a relation between the surface stress with respect to the chemical potential can be obtained as

$$
\frac{\partial \boldsymbol{\tau}}{\partial \mu_{i}}=-\Gamma_{i} \boldsymbol{I}-\frac{\partial \Gamma_{i}}{\partial \boldsymbol{\varepsilon}}
$$

Given the relationship between $\Gamma_{i}$ and $\mu_{i}$, we can calculate the dependence of the surface stress on adsorption from the Eq. (4).

For the single species localized adsorption at low coverage, the interaction between the adsorbates is neglected and the canonical partition function for the adsorbed atoms is

$$
Z_{a}=\sum_{i} \exp \left(-\frac{E_{i}}{k_{B} T}\right)
$$

where $E_{i}$ is the adsorption energy for one atom adsorbing on the surface, and $k_{B}$ is the Boltzmann constant, $T$ is the temperature. For $N_{a}$ adsorbed atoms over $N_{s}$ adsorption sites with the same adsorption energy $E_{a}$, the partition function can be written as

$$
Z=Q\left(N_{a}, N_{s}\right) q^{N_{a}} \exp \left(-\frac{N_{a} E_{a}}{k_{B} T}\right),
$$

where $Q$ denotes the distinguishable configurations and $q$ is the single particle partition function. At low coverage, there are many ways to arrange the adatoms on the available adsorption sites, which produce the configurational entropy. $Q$ is defined as [32]

$$
Q\left(N_{a}, N_{s}\right)=\frac{N_{s} !}{\left(N_{a} !\right)\left(N_{s}-N_{a}\right) !} .
$$


In consequence, the surface free energy $F_{s}$ is obtained as

$$
\begin{aligned}
F_{s} & =-k_{B} T \ln \left(Z_{a}\right) \\
& =-k_{B} T \ln \left[\frac{N_{s} ! q^{N_{a}}}{\left(N_{a} !\right)\left(N_{s}-N_{a}\right) !} \exp \left(-\frac{N_{a} E_{a}}{k_{B} T}\right)\right],
\end{aligned}
$$

With the Stirling's approximation ( $N \gg 1$ ), $\ln (N !) \approx N \ln N-N$, the formula above can be reduced to

$$
\begin{aligned}
F_{s} & =-k_{B} T N_{s} \ln N_{s}+k_{B} T N_{a} \ln N_{a} \\
& +k_{B} T\left(N_{s}-N_{a}\right) \ln \left(N_{s}-N_{a}\right) \\
& +N_{a} E_{a}-k_{B} T N_{a} \ln q .
\end{aligned}
$$

An assumption is made that in submonolayer with no penetration of the adsorbing atoms into the bulk of the material. The total differential of the Helmholtz free energy of the surface can be written as [18]

$$
d F_{s}=-S_{s} d T+A \tau d \varepsilon+\mu d N_{a}
$$

For the isothermal processes on an isotropic surface, the first term in the right of the Eq. (10) disappears. The chemical potential on the surface can be expressed as

$$
\mu=\frac{\partial F_{s}}{\partial N_{a}}=k_{B} T \ln \left(\frac{\theta}{1-\theta}\right)+E_{a}-k_{B} T \ln q
$$

in which, $\theta=N_{a} / N_{s}=a^{2} N_{a} / A$ (see Fig. 2). The first term on the right-hand side of Eq. (11) is the configurational contribution in terms of the adatom coverage $\theta$, the second term is the adsorption energy, and the last term is the vibrational contribution. So the Langmuir isotherm can be used to relate the surface coverage to the gas pressure by

$$
\frac{\theta}{1-\theta}=P q \exp \left(-\frac{E_{a}}{k_{B} T}\right),
$$

where $P$ is the pressure. Eriksson [33] and Halsey [34] have proved the relationship between the stress and strain in Eqs. (2) and (4) can be decoupled for approximation. So Eq. (2) can be integrated from $P=0$ to $P[30,34]$ for constant temperature and strain, combing the Eqs. (11) and (12), which can give for surface energy change

$$
\Delta \gamma=\frac{k_{B} T}{a^{2}} \ln (1-\theta)
$$

And surface stress change $\Delta \tau$ on the isotropic surface can be integrated from the Eq. (4)

$$
\Delta \tau=\frac{k_{B} T}{a^{2}} \ln (1-\theta)+k_{B} T \frac{\partial}{\partial \varepsilon}\left[\frac{1}{a^{2}} \ln (1-\theta)\right]
$$

Considering Eqs. (12) and (14), we can obtain

$$
\Delta \tau=\frac{k_{B} T}{a^{2}} \ln (1-\theta)+\frac{\theta}{a^{2}} \frac{\partial E_{a}}{\partial \varepsilon} .
$$

For simplification, the entropy change with strain is neglected in the above expression. The surface stress change $\Delta \tau$ is proportional to the natural logarithm of the coverage $\theta$ and to the adsorption energy change with strain. Based on the data of the $a b$ initio calculation [29], we suppose the relationship between the adsorption energy and the infinitesimal strain as

$$
E_{a}=C_{1}+C_{2} \mathcal{E}
$$

where $C_{1}, C_{2}$ are constants with the dimension of $\mathrm{eV}$, which can be determined by the experiments or fitted from the calculations. Substituting Eq. (16) into the Eq. (17), we have

$$
\Delta \tau=\frac{k_{B} T}{a^{2}} \ln (1-\theta)+\frac{C_{2}}{a^{2}} \theta .
$$

With $\tau_{0}=k_{B} T / a^{2}$, the formula above can be nondimensionlized into 


$$
\bar{\tau}=\frac{\Delta \tau}{\tau_{0}}=\ln (1-\theta)+\frac{C_{2}}{k_{B} T} \theta
$$

\section{Adsorabte-adsorbate interaction}

The interactions between adsorbates on solid surface can be classified as direct or indirect one, which means the adsorbates interact with each other directly or indirectly through the substrate [20,32]. Direct interactions occur when the adsorbates separation is small enough (high coverage adsorption), for example, dipole-dipole interactions. Elastic interactions arise when an adsorbate binds to a surface and perturbs the positions of the surrounding atoms. When a second atom adsorbs onto the surface, its binding energy is changed. Lau and Kohn [10] performed the first extensive analysis of elastic interactions. Based on continuum elasticity theory, Lau et al. found that the interaction between two like adatoms on an anisotropic substrate can be attractive in certain directions and repulsive in other.

The direct repulsive (Fig. 3(a)) or attractive (Fig. 3(b)) interactions between adsorbates as well as dipolar interactions contribute little to the induced surface stress. The direct repulsive interaction contributes to the surface stress is about $15 \%$ of the experiment value for the oxygen adsorption on $\mathrm{Ni}(111)$ [35]. In the physisorption layer, we adopt the Lennard-Jones (L-J) potential to describe the interaction between the adsorbates. For the L-J potential

$$
U\left(r_{i j}\right)=\varepsilon\left[\left(\frac{r_{0}}{r_{i j}}\right)^{12}-2\left(\frac{r_{0}}{r_{i j}}\right)^{6}\right],
$$

where $\varepsilon$ is the depth of the potential energy minimum and $r_{0}$ is the van der Waals diameter of the adsorbed atoms, $r_{i j}$ is the distance between the adsorbed atom $i$ and $j$. The surface stress associated with the adsorbates interactions is calculated as [35]

$$
\tau=-\frac{r_{i j}}{a^{2}} \frac{\partial U\left(r_{i j}\right)}{\partial r_{i j}}=\frac{12 \varepsilon}{a^{2}}\left[\left(\frac{r_{0}}{r_{i j}}\right)^{12}-\left(\frac{r_{0}}{r_{i j}}\right)^{6}\right]
$$

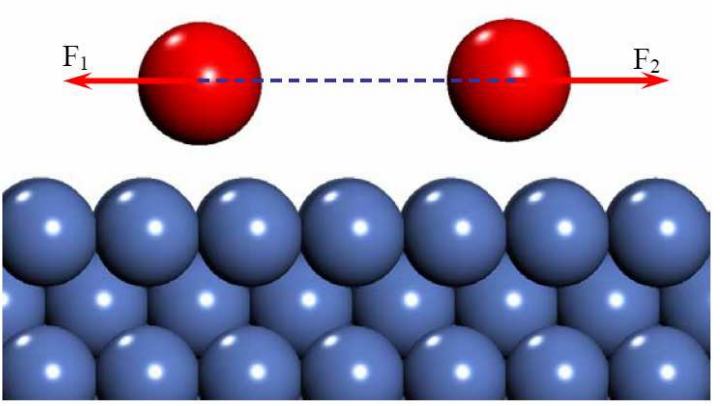

(a)

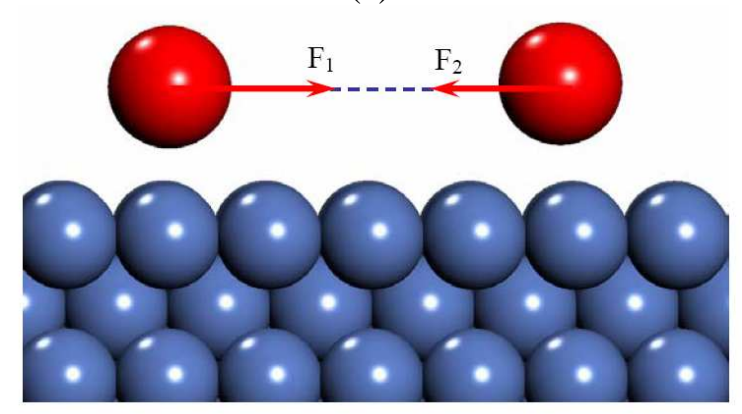

(b)
Figure 3. The interaction between the adsorbates. (a) Repulsive interaction; (b) Attractive interaction.

Introducing $\tau_{0}=12 \varepsilon / a^{2}$ and $\bar{r}=r_{i j} / r_{0}$, this equation can be nondimensionalized to

$$
\bar{\tau}=(\bar{r})^{-12}-(\bar{r})^{-6} .
$$

The substrate-mediated interaction is the indirect interaction between the adsorbates. For the physisorption, a simple expression of the substrate-mediated energy is Sinanoglu-PitzerMcLachlan (SPM) energy [19,20,36,37]

$$
U\left(r_{i j}\right)=\frac{9 \alpha}{16} \frac{S}{L^{3}} \frac{1}{r_{i j}^{3}}
$$

where $\alpha$ is the adatom polarizability $\left(\AA^{3}\right), S$ is the strength coefficient of the adatomsubstrate interactions $\left(\mathrm{eV} \cdot \AA^{3}\right), L$ is the perpendicular distance of the adatom from the 
substrate $(\AA)$. So the substrate-mediated interaction induced surface stress can be obtained as

$$
\tau=\frac{27 \alpha}{16 a^{2}} \frac{S}{L^{3}} \frac{1}{r_{i j}^{3}}
$$

which can also be nondimensionalized to

$$
\bar{\tau}=\frac{\tau}{\tau_{0}}=(\bar{r})^{-3}
$$

with $\tau_{0}=\frac{27 \alpha}{16 a^{2}} \frac{S}{\left(r_{0} L\right)^{3}}$. This formula can also be used in chemisorption with the modified parameters $[19,20]$.

\section{Results and discussion}

On the basis of the thermodynamics, we establish a theory model for the adsorption induced surface stress as Eq. (18). It is shown that the adsorption induced surface stress is determined by two factors. One is the adsorbate coverage and the other is the partial derivative of the adsorption energy with respect to the surface strain [8]. The relationship between the adsorption energy and the surface strain is generally decided by the properties of the interaction as well as the properties of the surfaces. Atomic simulation may be a convenient method to give the details of the interaction between adsorbates and substrate. With $\beta=C_{2} N_{s} /\left(k_{B} T\right)$, the Eq. (18) is reduced to

$$
\bar{\tau}=\ln (1-\theta)+\beta \theta
$$

For $\beta \in[-2,3]$, the nondimensional adsorption induced surface stress changes with the adsorbates coverage is plotted (Fig. 4). For the all the values of $\beta$, the variation of the adsorption induced surface stress with coverage is nonlinear, and the curves exhibits a larger nonlinear characters for smaller absolute value of $\beta$. Some qualitative discussion of the sign of the surface stress change $\Delta \tau$ has been reported by Ibach [17] for transition and noble metals. Indeed, since such metals have a tensile surface stress $(\tau>0)$, electronegative adsorbates removes charges between the surface atoms and thus reduces the surface stress which may become negative (compressive). On the contrary, if the substrate is electronegative with respect to the adsorbates, the surface stress may increase [18].

The effects of the adsorbate-adsorbate interaction on the surface stress are rather small for the low coverage, but it may be considerable in the high coverage. Based on the L-J potential and SPM energy, the contributions to the surface stress by the adsorbate-adsorbate interactions are deduced as Eqs. (21) and (24). The nondimensional surface stresses are plotted with respected to the distance between the adsorbates (Fig. 5). For the direct interaction, when $\bar{r} \rightarrow \infty$, the interactions between the adsorbates do not contribute to the surface stress. For $\bar{r}<1$, the interactions give tensile surface stress, and compressive surface stress for $\bar{r}>1$. While for the indirect interaction of substrate-mediated interaction, the contribution is always tensile surface stress in the frame of SPM energy and the surface stress changes as $\bar{r}^{3}$. The adsorption induced surface stress is compressive when the adsorbates are pulling on another apart, or be tensile when the surface adsorbates are pushing on another.

For the high coverage, the L-J potential is used to describe the interaction between the adsorbates and the total adsorption induced surface stress can be obtained as

$$
\begin{aligned}
\Delta \tau_{\text {tot }}=\Delta \tau_{\text {adsorption }}+\Delta \tau_{\text {interaction }} \\
=\frac{k_{B} T}{a^{2}} \ln (1-\theta)+\frac{C_{2}}{a^{2}} \theta \\
+\frac{12 \varepsilon_{0}}{a^{2}}\left[\left(\frac{r_{0}}{r_{i j}}\right)^{12}-\left(\frac{r_{0}}{r_{i j}}\right)^{6}\right] .
\end{aligned}
$$

With $\Delta \bar{\tau}_{\text {tot }}=\Delta \tau_{\text {tot }} / \tau_{0}, \bar{r}=r_{i j} / r_{0}$ and $\zeta=12 \varepsilon_{0} /\left(k_{B} T\right)$, the formula (26) should be reduced to 


$$
\Delta \bar{\tau}_{\mathrm{tot}}=\ln (1-\theta)+\beta \theta+\zeta\left[(\bar{r})^{-12}-(\bar{r})^{-6}\right] .
$$

Considering the carbon atoms adsorption on the $\mathrm{Ni}(100)$ surface, the L-J parameters of carbon atoms is $\varepsilon_{0}=-0.11 \mathrm{kcal} / \mathrm{mol}, r_{0}=2 \AA$. The lattice constant of $\mathrm{Ni}$ is $3.524 \AA$. When $\theta$ equals to 1 , the distance between adatoms is $r_{\min }$. For arbitrary $\theta, r_{i j}=r_{\min } \theta^{-1 / 2}$. In the situation of $\beta=-0.5$, the total adsorption induced surface stress can be obtained as Fig. 6, based on Eq. (27). It is demonstrated that it is reasonable to neglect the interaction between the adsorbates for low coverage. While for high coverage $(\theta>0.5)$, the interaction between adsorbates show obvious effects on the adsorption induced surface.

\section{Conclusion}

A model of the adsorption induced surface stress has been established for the low coverage based on the statistical thermodynamics theory. The adsorption induced surface stress was found to relate to adsorbates coverage and the partial derivative of the adsorption energy with respect to the surface strain. Due to the properties of the adsorption, the adsorption induced surface stress can be compressive or tensile. For the high coverage, the interactions between the adsorbate also contribute to the surface stress.

The normal deformation of the elastic surface is not considered in this paper, which is worth more theoretical and experimental researches, especially on adsorption induced surface relaxation and reconstruction.

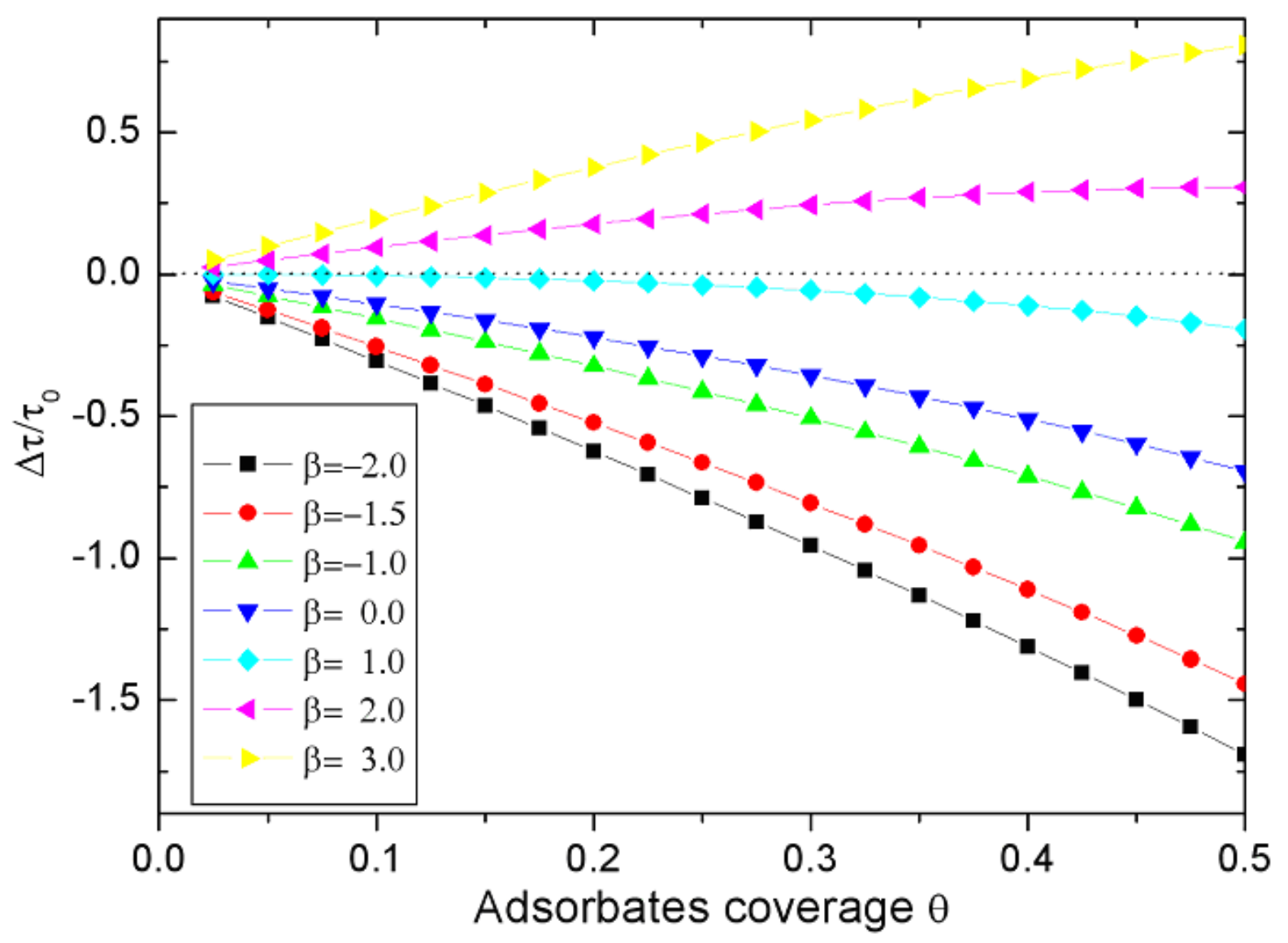

Figure 4. Plots of nondimensional surface stress versus adsorbates coverage for the parameter $\beta$. 


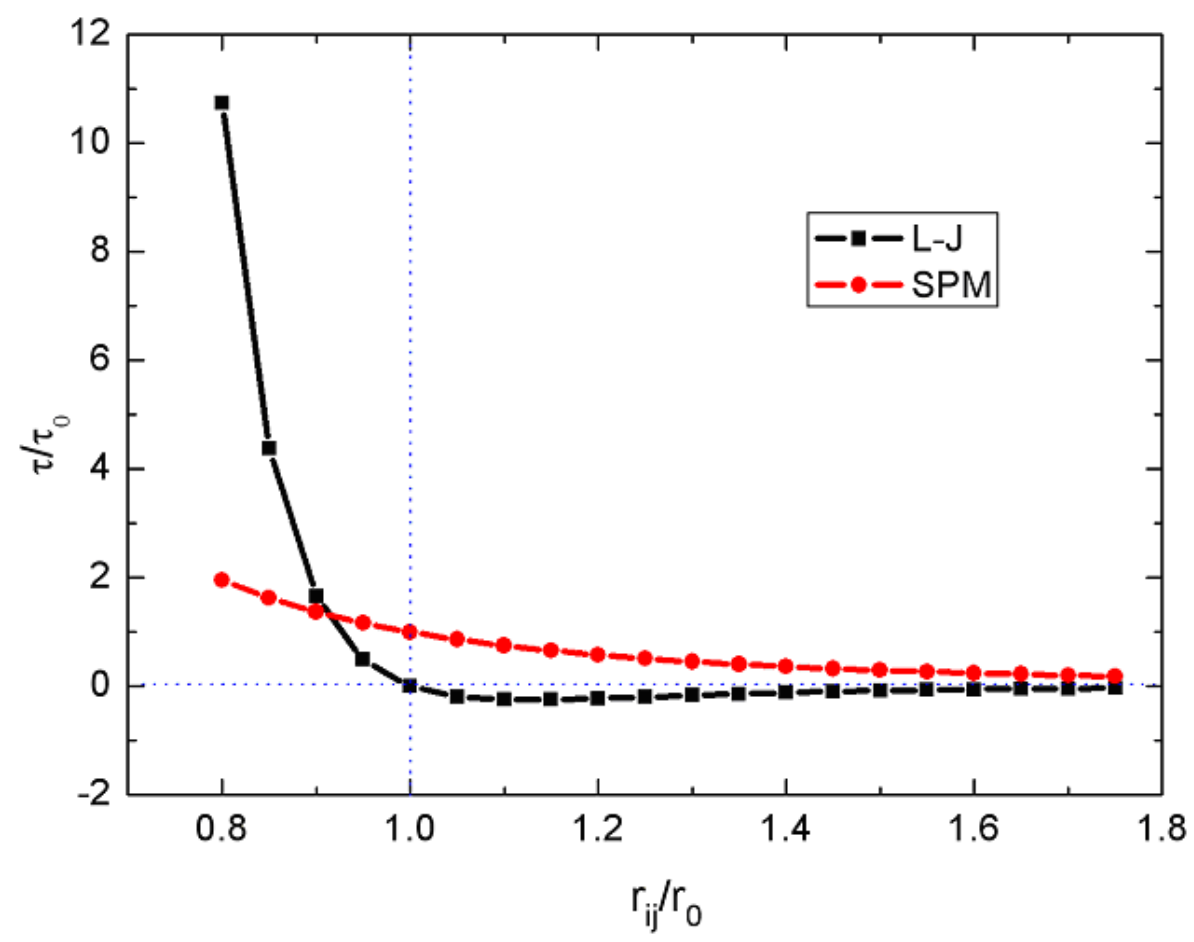

Figure 5. The nondimensional surface stress induced by the interaction between the adsorbates.

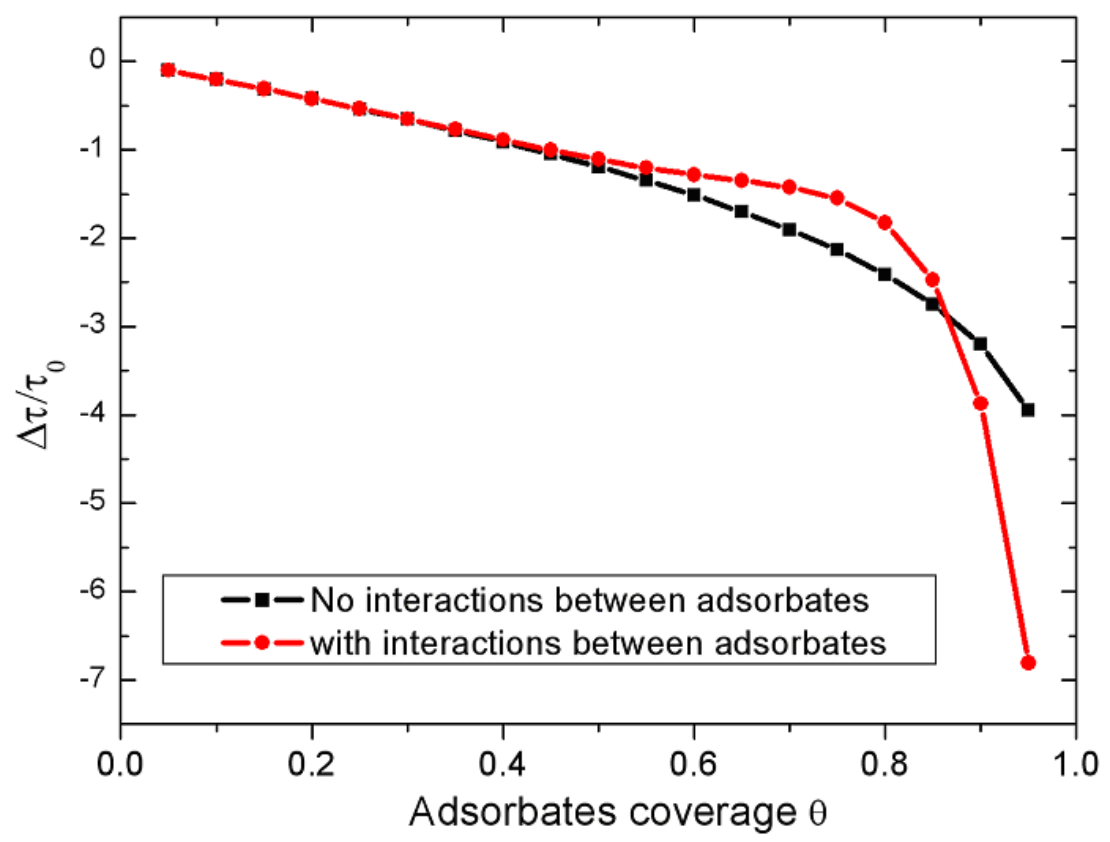

Figure 6. The effects of the interaction between adsorbates on adsorption-induced surface stress.

\section{Acknowledgments}

This research was jointly supported by the National Basic Research Program of China (973 Program, Grant No. 2007CB310500), the
National High-tech R\&D Program of China (863 Program, Grant No. 2007AA04Z348) and the National Natural Science Foundation of China (NSFC, Grant No. 10772180). 


\section{References}

[1] Berger R, Delamarche E, Lang HP. Surface stress in the self-assembly of alkanethiols on gold, Science, 276: 2021-2024 (1997)

[2] Sarikaya M, Tamerler C, Jen AKY, Schulten K, Baneyx F. Molecular biomimetics: nanotechnology through biology, Nature Mat, 2: 577-585 (2003)

[3] Ren Q, Zhao YP. Influence of surface stress on frequency of microcantilever-based biosensors. Microsyst Technol, 10:307-314 (2004).

[4] Huang GY, Gao W, Yu SW. Model for the adsorption-induced change in resonance frequency of a cantilever. Appl Phys Lett, 89: 043506 (2006).

[5] Ren Q, Zhao YP, Han L, Zhao HB. A nanomechanical device based on light-driven proton pumps, Nanotechnology, 17:1778-1785 (2006)

[6] Schell-Sorokin AJ, Tromp RM. Mechanical stresses in (sub)monolayer epitaxial films, Phys Rev Lett, 64: 1039-1042 (1990)

[7] Sander D, Ibach H. Experimental determination of adsorbate-induced surface stress: oxygen on $\mathrm{Si}(111)$ and $\mathrm{Si}(100)$. Phys Rev B, 45: 4263-4267 (1991)

[8] Sander D, Linke U, Ibach $H$. Adsorbate-induced stress: surface, oxygen and carbon on $\mathrm{Ni}(100)$, Surf Sci, 272: 318-325 (1992)

[9] Kohn W, Lau KH. Adatom dipole moments on metals and their interaction, Solid State Commun, 18: 553-555 (1976)

[10] Lau KH, Kohn W. Elastic interaction of two atoms adsorbed on a solid surface, Surf Sci, 65: 607-618 (1977)

[11] Kukta RV, Kouris D, Sieradzki K. Adatoms and their relation to surface stress, $J$ Mech Phys Solid, 51: 1243-1266 (2003)

[12] Kukta RV, Liu P, Kouris D. On the dependence of adatom interactions on strain, J Mech Phys Solid, 51: 2149-2167 (2003)

[13] Pala RGS, Liu F. Determining the adsorptive and catalytic properties of strained metal surfaces using adsorption-induced stress, J Chem Phys, 120: 7720-7724 (2004)
[14] Levi AC, Pisoni C. Surface deformation in molecular physisorption, Surf Sci, 566: 683-687 (2004)

[15] Calvini P, Levi AC. Elastic theory of surface deformation in molecular physisorption, Surf Sci, 594: 43-53 (2005)

[16] He LH, Lim CW. Surface Green function for a soft elastic half-space: Influence of surface stress, Inter J Solids Struct, 43: 132-143 (2006)

[17] Ibach H. Adsorbate-induced surface stress, J Vac Sci Technol A, 12:2240-2245 (1994)

[18] Ibach H. The relation between the strain-dependence of the heat of adsorption and the coverage dependence of the adsorbate induced surface stress, Surf Sci, 556: 71-77 (2004)

[19] Wang XQ, Chen R, Wang YL, He TJ, Liu FC. Theoretical studies on electrocompression of electrodeposited halide monolayer on $\operatorname{Au}(111)$ surface, $J$ Phys Chem B, 102: 7568-7576 (1998)

[20] Xie R, Chen DM, Wang XQ, He TJ, Liu FC. Adsorbate-induced surface stress of chloride monolayer on $\mathrm{Au}(111)$ electrode, $J$ Phys Chem B, 106: 12948-12956 (2002)

[21] Brako R, Šokčević D. Adsorbate-induced substrate relaxation and the adsorbate-adsorbate interaction, Surf Sci, 469: 185-195 (2000)

[22] Brako R, Šokčević D. Adsorbate-adsorbate interaction mediated by substrate lattice, Surf Sci, 454: 623-627 (2000)

[23] Xu L, He JH, Liu Y T. Electrospun nanoporous spheres with Chinese drug, Int $J$ Nonlinear Sci Numer Simul, 8: 199-202 (2007)

[24] He JH, Wan YQ, Xu L. Nano-effects, quantum-like properties in electrospun nanofibers, Chaos Solitons \& Fractals, 33: 26-37 (2007)

[25] Chen X, Cui DF, Wang L, Wang M, Zhao Q. Development and characterization of DNA hybridization reaction on PDMS microchip, Int J Nonlinear Sci Numer Simul, 3: 211-214 (2002)

[26] Oscarsson S. Surface functionalisation, orientation, conformation and positioning of macromolecules at the nanometer scale, Int 
J Nonlinear Sci Numer Simul, 3: 747-749 (2002)

[27] Dai W, Zhao YP. The nanolinear phenomena of thin polydimethylsiloxane (PDMS) films in electrowetting, Int $J$ Nonlinear Sci Numer Simul, 8: 519-526 (2007)

[28] Lin WH, Zhao YP. Pull-in instability of micro-switch actuators: Model review, Int $J$ Nonlinear Sci Numer Simul, 9: 175-183 (2008)

[29] Hong SY, Kara A, Rahman TS, Heid R, Bohnen KP. $A b$ initio calculations of adsorbate-induced stress on $\mathrm{Ni}(100)$, Phys Rev B, 69: 195403 (2004)

[30] Müller P, Saúl A. Elastic effects on surface physics, Surf Sci Rep, 54: 157-258 (2004)

[31] Shuttleworth R. The surface tension of solid, Proc Phys Soc A, 163: 444-457 (1950)
[32] Hill TL. An Introduction to Statistical Thermodynamics, Dove, New York, 1986

[33] Eriksson JC. Thermodynamics of surface phase systems v. Contribution to the thermodynamics of the solid-gas interface, Surf Sci, 14: 221-246 (1969)

[34] Halsey GD. Surface tension and spreading pressure of mobile and registered monolayers, Surf Sci, 72: 1-71 (1978)

[35] Ibach H. The role of surface stress in reconstruction, epitaxial growth and stabilization of mesoscopic structures, Surf Sci Rep, 29: 193-263 (1997)

[36] Price GL, Venables JA. A theory of the phase transitions in solid krypton and xenon monolayers on graphite, Surf Sci, 59: 509-532 (1976)

[37] Bruch LW. Theory of physisorption interactions, Surf Sci, 125: 194-217 (1983) 